\title{
Comparison of Optimal Homotopy Asymptotic and Adomian Decomposition Methods for a Thin Film Flow of a Third Grade Fluid on a Moving Belt
}

\author{
Fazle Mabood $^{1}$ and Nopparat Pochai ${ }^{2,3}$ \\ ${ }^{1}$ Department of Mathematics, University of Peshawar, Peshawar, Pakistan \\ ${ }^{2}$ Department of Mathematics, King Mongkut's Institute of Technology Ladkrabang, Bangkok 10520, Thailand \\ ${ }^{3}$ Centre of Excellence in Mathematics, CHE, Si Ayutthaya Road, Bangkok 10400, Thailand \\ Correspondence should be addressed to Nopparat Pochai; nop_math@yahoo.com
}

Received 24 March 2015; Accepted 11 May 2015

Academic Editor: John D. Clayton

Copyright (C) 2015 F. Mabood and N. Pochai. This is an open access article distributed under the Creative Commons Attribution License, which permits unrestricted use, distribution, and reproduction in any medium, provided the original work is properly cited.

We have investigated a thin film flow of a third grade fluid on a moving belt using a powerful and relatively new approximate analytical technique known as optimal homotopy asymptotic method (OHAM). The variation of velocity profile for different parameters is compared with the numerical values obtained by Runge-Kutta Fehlberg fourth-fifth order method and with Adomian Decomposition Method (ADM). An interesting result of the analysis is that the three terms OHAM solution is more accurate than five terms of the ADM solution and this thus confirms the feasibility of the proposed method.

\section{Introduction}

Many physical systems in fluid mechanics generally lead to nonlinear ordinary or partial differential equations. Due to complexity of Non-Newtonian fluid, it is difficult to solve nonlinear differential equation. A second grade fluid is one of the most acceptable fluids in this sub clam of Non-Newtonian fluids, because of its mathematical simplicity in comparison to third grade and fourth grade fluids. In related literature many authors have effectively treated the complicated nonlinear equations governing the flow of a third grade fluid $[1,2]$.

Since the Non-Newtonian fluids are of the great challenges in the solution of governing nonlinear differential equations, many numerical and analytical techniques have been proposed by many researchers. But an efficient approximate analytical solution still finds enormous appreciations. Keeping this fact in mind, we have solved the governing nonlinear equation of the present problem using the two techniques.
It is important to mention here that the analytical and numerical solutions are in a good agreement but better than the results of Siddiqui et al. [3].

In this study, it is also observed that the optimal homotopy asymptotic method is a powerful approximate analytical tool that is simple and straightforward and does not require the existence of any small or large parameter as does traditional perturbation method. Optimal homotopy asymptotic method has successfully been applied to a number of nonlinear problems arising in the science and engineering by various researchers [4-9]. This proves the validity and acceptability of OHAM as a useful solution technique.

This paper is organized as follows. First in Section 2, we formulate the problem. In Section 3 we present basic principles of OHAM. The OHAM solution is given in Section 4. In Section 5, we analyze the comparison of the solution using OHAM with existing solution of ADM. Section 6 is devoted for the conclusion. 


\section{Governing Equation}

The thin film flow of third grade fluid on a moving belt is governed by the following nonlinear boundary value problem [3]:

$$
\begin{aligned}
\frac{d^{2} v}{d x^{2}}+\frac{6\left(\beta_{2}+\beta_{3}\right)}{\mu}\left(\frac{d v}{d x}\right)^{2} \frac{d^{2} v}{d x^{2}}-\frac{\rho g}{\mu} & =0, \\
v(0) & =U_{0}, \\
\frac{d v}{d x} & =0 \text { at } x=\delta,
\end{aligned}
$$

where $v$ is the fluid velocity, $\rho$ is the density, $\mu$ is the dynamic viscosity, $\beta_{2}$ and $\beta_{3}$ are the material constants of the third grade fluid, $g$ is acceleration due to gravity, $\delta$ is the uniform thickness of the fluid film, and $U_{0}$ is the speed of the belt.

Here, we introduce the following dimensionless variables:

$$
\begin{aligned}
x^{*} & =\frac{x}{\delta}, \\
v^{*} & =\frac{v}{U_{0}}, \\
\beta & =\frac{\left(\beta_{2}+\beta_{3}\right)}{\mu \delta^{2}} U_{0}, \\
m & =\frac{\rho g}{\mu U_{0}} \delta^{2} .
\end{aligned}
$$

From (1)-(2), we obtain the dimensionless form as

(for simplicity we removed $*$ )

$$
\frac{d^{2} v}{d x^{2}}+6 \beta\left(\frac{d v}{d x}\right)^{2} \frac{d^{2} v}{d x^{2}}-m=0,
$$

subject to the boundary conditions

$$
\begin{gathered}
v(0)=1, \\
v^{\prime}(1)=0 .
\end{gathered}
$$

\section{Optimal Homotopy Asymptotic Method}

We review the basic principles of OHAM as expounded in [4-8] in five steps.

(i) Let us consider the following differential equation:

$$
A[v(\tau)]+a(\tau)=0, \quad \tau \in \Omega,
$$

where $\Omega$ is problem domain, $A(v)=L(v)+N(v)$, where $L, N$ are linear and nonlinear operator, $v(\tau)$ is an unknown function, and $a(\tau)$ is a known function.

(ii) Construct an optimal homotopy equation as

$$
\begin{aligned}
& (1-p)[L(\phi(\tau ; p))+a(\tau)] \\
& -H(p)[A(\phi(\tau ; p))+a(\tau)]=0,
\end{aligned}
$$

where $0 \leq p \leq 1$ is an embedding parameter and $H(p)=$ $\sum_{k=1}^{m} p^{k} C_{k}$ is auxiliary function on which the convergence of the solution greatly depends. The auxiliary function $H(p)$ also adjust the convergence domain and control the convergence region. According to the new development in OHAM [9] the more generalized form of the auxiliary function is $H\left(\tau ; p, C_{i}\right)=p H_{1}\left(\tau, C_{i}\right)+p^{2} H_{2}\left(\tau, C_{i}\right)+\ldots$, where $H_{i}\left(\tau, C_{i}\right)$, $i=1,2, \ldots$ are auxiliary functions depending upon $\tau$ and unknown parameters $C_{j}$. This means that we could have more convergence-control parameters even at the first order of approximation.

(iii) Expand $\phi\left(\tau ; p, C_{j}\right)$ in Taylor's series about $p$; one has an approximate solution:

$$
\begin{aligned}
& \phi\left(\tau ; p, C_{j}\right)=v_{0}(\tau)+\sum_{k=1}^{\infty} v_{k}\left(\tau, C_{j}\right) p^{k}, \\
& j=1,2,3, \ldots
\end{aligned}
$$

Many researchers have observed that the convergence of the series (7) depends upon $C_{j},(j=1,2, \ldots, m)$, if it is convergent then, we obtain

$$
\widetilde{v}=v_{0}(\tau)+\sum_{k=1}^{m} v_{k}\left(\tau ; C_{j}\right) .
$$

(iv) Substituting (8) in (6), we have the following residual:

$$
R\left(\tau ; C_{j}\right)=L\left(\widetilde{v}\left(\tau ; C_{j}\right)\right)+a(\tau)+N\left(\widetilde{v}\left(\tau ; C_{j}\right)\right) .
$$

If $R\left(\tau ; C_{j}\right)=0$, then $\widetilde{v}$ will be the exact solution. For nonlinear problems, generally this will not be the case. For determining $C_{j},(j=1,2, \ldots, m)$, Galerkin's Method, Ritz Method, or the method of least squares can be used.

(v) Finally, substitute these constants in (8) and one can get the approximate solution.

\section{Solution of the Problem via OHAM}

According to the OHAM, applying (6) to (3)

$$
(1-p)\left(v^{\prime \prime}\right)-H(p)\left\{v^{\prime \prime}+6 \beta v^{\prime 2} v^{\prime \prime}-m\right\}=0,
$$

where primes denote differentiation with respect to $x$.

We consider $v$ and $H(p)$ as the following:

$$
\begin{aligned}
v & =v_{0}+p v_{1}+p^{2} v_{2}, \\
H(p) & =p C_{1}+p^{2} C_{2} .
\end{aligned}
$$

Put (11) in (10) and arrange the terms according to the powers of $\mathrm{p}$ to get zeroth, first, and second order problems as follows.

Zeroth order problem is

$$
v_{0}^{\prime \prime}(x)=m
$$

with boundary conditions

$$
\begin{aligned}
& v_{0}(0)=1, \\
& v_{0}^{\prime}(1)=0 .
\end{aligned}
$$


Its solution is

$$
v_{0}=\frac{1}{2}\left(2-2 m x+m x^{2}\right)
$$

First order problem is

$$
\begin{aligned}
v_{1}^{\prime \prime}\left(x, C_{1}\right)= & -m-m C_{1}+6 \beta C_{1}\left(v_{0}^{\prime}\right)^{2} v_{0}^{\prime \prime} \\
& +\left(1+C_{1}\right) v_{0}^{\prime \prime}(x)
\end{aligned}
$$

with boundary conditions

$$
\begin{aligned}
& v_{1}(0)=0, \\
& v_{1}^{\prime}(1)=0,
\end{aligned}
$$

having solution

$$
\begin{aligned}
& v_{1}\left(x, C_{1}\right)=\frac{1}{2}\left(-4 m^{3} \beta x C_{1}+6 m^{3} \beta x^{2} C_{1}\right. \\
& \left.-4 m^{3} \beta x^{3} C_{1}+m^{3} \beta x^{4} C_{1}\right) .
\end{aligned}
$$

Second order problem is

$$
\begin{aligned}
v_{2}^{\prime \prime}\left(x, C_{1}, C_{2}\right)= & -m C_{2}+C_{2} v_{0}^{\prime \prime}+6 \beta C_{2}\left(v_{0}^{\prime}\right)^{2} v_{0}^{\prime \prime} \\
& +12 \beta C_{1} v_{0}^{\prime} v_{1}^{\prime} v_{0}^{\prime \prime}+6 \beta C_{1}\left(v_{0}^{\prime}\right)^{2} v_{1}^{\prime \prime} \\
& +\left(1+C_{1}\right) v_{1}^{\prime \prime}
\end{aligned}
$$

with boundary conditions

$$
\begin{aligned}
& v_{2}(0)=0, \\
& v_{2}^{\prime}(1)=0 .
\end{aligned}
$$

Its solution becomes

$$
\begin{aligned}
& v_{2}\left(x, C_{1}, C_{2}\right)=\frac{1}{2}\left(-4 m^{3} \beta x C_{1}+6 m^{3} \beta x^{2} C_{1}\right. \\
& \quad-4 m^{3} \beta x^{3} C_{1}+m^{3} \beta x^{4} C_{1}-4 m^{3} \beta x C_{1}{ }^{2} \\
& \quad-24 m^{5} \beta^{2} x C_{1}{ }^{2}+6 m^{3} \beta x^{2} C_{1}{ }^{2}+60 m^{5} \beta^{2} x^{2} C_{1}{ }^{2} \\
& -4 m^{3} \beta x^{3} C_{1}{ }^{2}-80 m^{5} \beta^{2} x^{3} C_{1}{ }^{2}+m^{3} \beta x^{4} C_{1}{ }^{2} \\
& +60 m^{5} \beta^{2} x^{4} C_{1}{ }^{2}-24 m^{5} \beta^{2} x^{5} C_{1}{ }^{2}+4 m^{5} \beta^{2} x^{6} C_{1}{ }^{2} \\
& \quad-4 m^{3} \beta x C_{2}+6 m^{3} \beta x^{2} C_{2}-4 m^{3} \beta x^{3} C_{2} \\
& \left.+m^{3} \beta x^{4} C_{2}\right) .
\end{aligned}
$$

We obtain the three terms solution using OHAM for $p=1$

$$
\widetilde{v}\left(x, C_{1}, C_{2}\right)=v_{0}(x)+v_{1}\left(x, C_{1}\right)+v_{2}\left(x, C_{1}, C_{2}\right) .
$$

From least squares method we obtain the unknown convergent constants $C_{1}, C_{2}$ in (21).

For the particular case, if $\beta=0.5$ and $m=0.2$, we have $C_{1}=-0.877411, C_{2}=-0.003097$.
TABLE 1: Comparison of absolute error using OHAM (three terms) and $\mathrm{ADM}$ (five terms) [3] for $\beta=0.5, m=0.5$.

\begin{tabular}{cccccc}
\hline$x$ & OHAM & ADM & NM & $\begin{array}{c}\text { Error } \\
(\mathrm{ADM})\end{array}$ & $\begin{array}{c}\text { Error } \\
(\text { OHAM })\end{array}$ \\
\hline 0.0 & 1 & 1 & 1 & 0 & 0 \\
0.1 & 0.959206 & 0.962523 & 0.959268 & $3.2 \times 10^{-3}$ & $6.2 \times 10^{-5}$ \\
0.2 & 0.921830 & 0.926065 & 0.9219695 & $4.1 \times 10^{-3}$ & $1.3 \times 10^{-4}$ \\
0.3 & 0.888047 & 0.892574 & 0.8882986 & $4.3 \times 10^{-3}$ & $2.5 \times 10^{-4}$ \\
0.4 & 0.858081 & 0.862771 & 0.8584654 & $4.3 \times 10^{-3}$ & $3.8 \times 10^{-4}$ \\
0.5 & 0.832177 & 0.836998 & 0.8326892 & $4.3 \times 10^{-3}$ & $5.1 \times 10^{-4}$ \\
0.6 & 0.810581 & 0.815502 & 0.8111930 & $4.3 \times 10^{-3}$ & $6.1 \times 10^{-4}$ \\
0.7 & 0.793520 & 0.798502 & 0.7941932 & $4.3 \times 10^{-3}$ & $6.7 \times 10^{-4}$ \\
0.8 & 0.781186 & 0.786196 & 0.7818867 & $4.3 \times 10^{-3}$ & $7.1 \times 10^{-4}$ \\
0.9 & 0.773724 & 0.778742 & 0.7744327 & $4.3 \times 10^{-3}$ & $7.1 \times 10^{-4}$ \\
1.0 & 0.771227 & 0.776245 & 0.7719358 & $4.3 \times 10^{-3}$ & $7.1 \times 10^{-4}$ \\
\hline
\end{tabular}

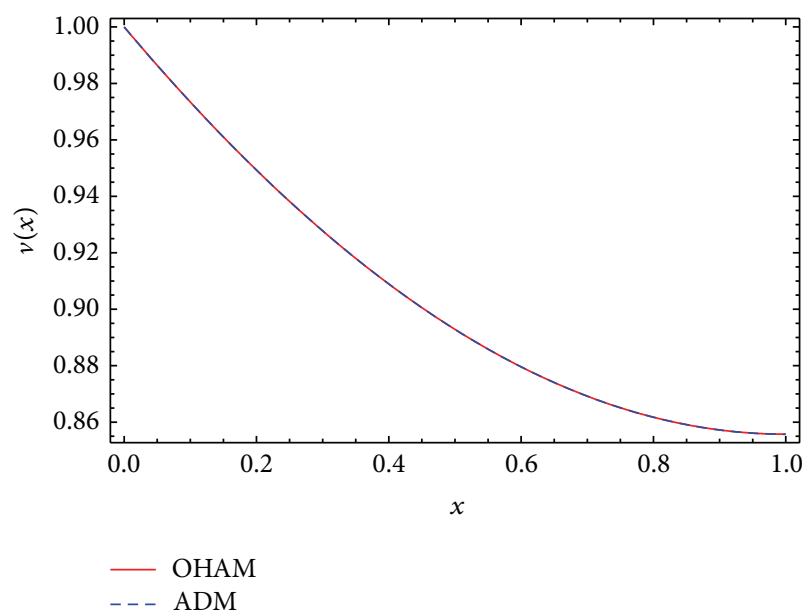

FIGURE 1: Comparison of velocity profile using OHAM (three terms) and ADM [3] (five terms) for $\beta=0.5, m=0.3$.

\section{Results and Discussion}

Table 1 shows the comparison of absolute error between OHAM (three terms) and ADM (five terms) [3]. It is noteworthy to mention here that OHAM low error is remarkable, while the effectiveness of the proposed method (OHAM) can be seen from Figure 1. The effect of fluid parameter $\beta$ is displayed in Figure 2. From Figure 2 it is found that the boundary layer thickness is increased with an increase in fluid parameter $\beta$ whereas the value of $m$ is preset. Whilst Figure 3 depicts an increase in $m$ for the fixed value of fluid parameter $\beta$, a decrease can be seen in boundary layer thickness. However, opposite observation is made by comparing of Figures 2 and 3.

\section{Conclusion}

Optimal homotopy asymptotic method is employed to investigate the approximate solution for a thin film flow of third grade fluid on a moving belt. Both numeric and analytic 


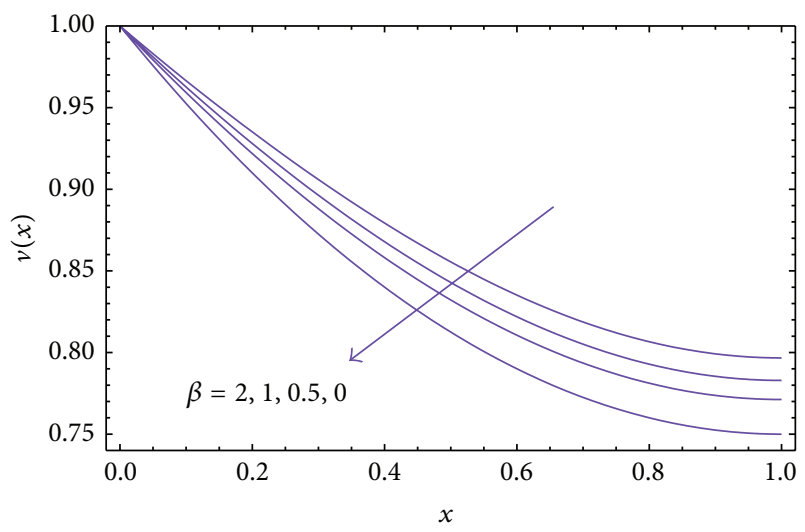

FIGURE 2: Effects on velocity profile for various values of $\beta$ at $m=$ 0.5 .

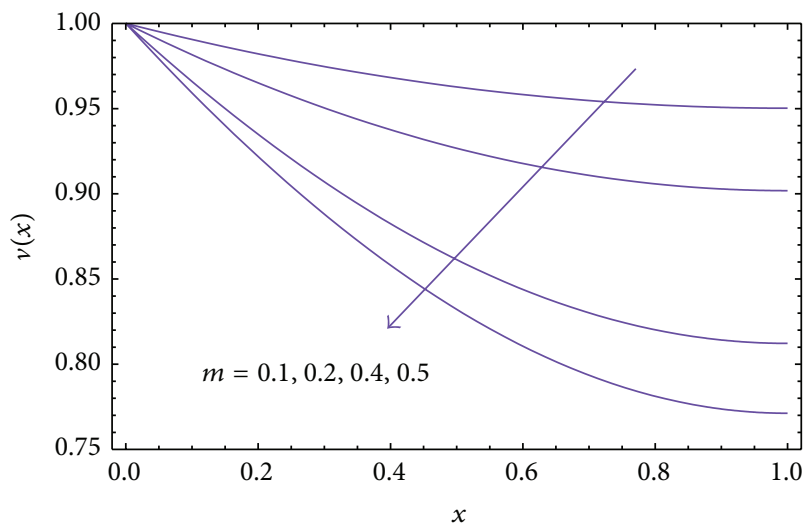

FIgURE 3: Effects on velocity profile for various values of $m$ at $\beta=$ 0.5 .

results are obtained for the problem. The results are sketched and discussed for the fluid parameter $\beta$ and for constant $m$. From the study it is revealed that the solution using OHAM is better than ADM results. Finally, we conclude that OHAM provide a simple and easy way to control and adjust the convergence region for strong nonlinearity and is applicable to highly nonlinear fluid problems.

\section{Conflict of Interests}

The authors declare no conflict of interests.

\section{Acknowledgments}

The second author appreciates the Center of Excellence in Mathematics, the Commission on Higher Education, Thailand. The authors greatly appreciate the valuable comments received from the referees.

\section{References}

[1] A. M. Siddiqui, R. Mahmood, and Q. K. Ghori, "Homotopy perturbation method for thin film flow of a third grade fluid down an inclined plane," Chaos, Solitons \& Fractals, vol. 35, no. 1, pp. 140-147, 2008.

[2] A. M. Siddiqui, R. Mahmood, and Q. K. Ghori, "Thin film flow of a third grade fluid on a moving belt by He's homotopy perturbation method," International Journal of Nonlinear Sciences and Numerical Simulation, vol. 7, no. 1, pp. 7-14, 2006.

[3] A. M. Siddiqui, A. A. Farooq, T. Haroon, and B. S. Babcock, "A comparison of variational iteration and Adomian decomposition methods in solving nonlinear thin film flow problems," Applied Mathematical Sciences, vol. 6, no. 97-100, pp. 4911-4919, 2012.

[4] N. Herişanu, V. Marinca, T. Dordea, and G. Madescu, "A new analytical approach to nonlinear vibration of an electrical machine," Proceedings of the Romanian Academy Series A: Mathematics Physics Technical Sciences Information Science, vol. 9, no. 3, pp. 229-236, 2008.

[5] V. Marinca and N. Herisanu, "Optimal homotopy perturbation method for strongly nonlinear differential equations," Nonlinear Science Letters A, vol. 1, no. 3, pp. 273-280, 2010.

[6] V. Marinca and N. Herişanu, "Application of optimal homotopy asymptotic method for solving nonlinear equations arising in heat transfer," International Communications in Heat and Mass Transfer, vol. 35, no. 6, pp. 710-715, 2008.

[7] F. Mabood, W. A. Khan, and A. I. M. Ismail, "Optimal homotopy asymptotic method for heat transfer in hollow sphere with robin boundary conditions," Heat Transfer-Asian Research, vol. 43, no. 2, pp. 124-133, 2014.

[8] V. Marinca and N. Herişanu, "Determination of periodic solutions for the motion of a particle on a rotating parabola by means of the optimal homotopy asymptotic method," Journal of Sound and Vibration, vol. 329, no. 9, pp. 1450-1459, 2010.

[9] N. Herisanu, V. Marinca, and G. Madescu, "An analytical approach to non-linear dynamical model of a permanent magnet synchronous generator," Wind Energy, 2014. 


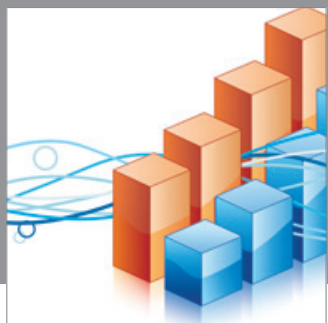

Advances in

Operations Research

mansans

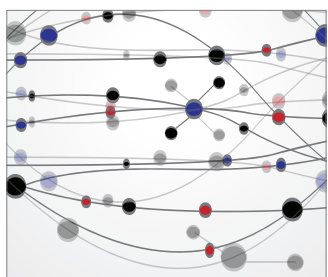

The Scientific World Journal
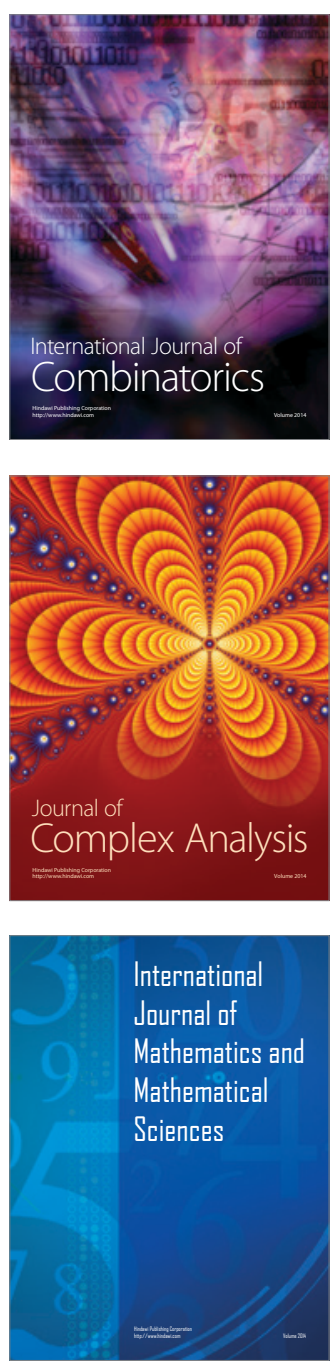
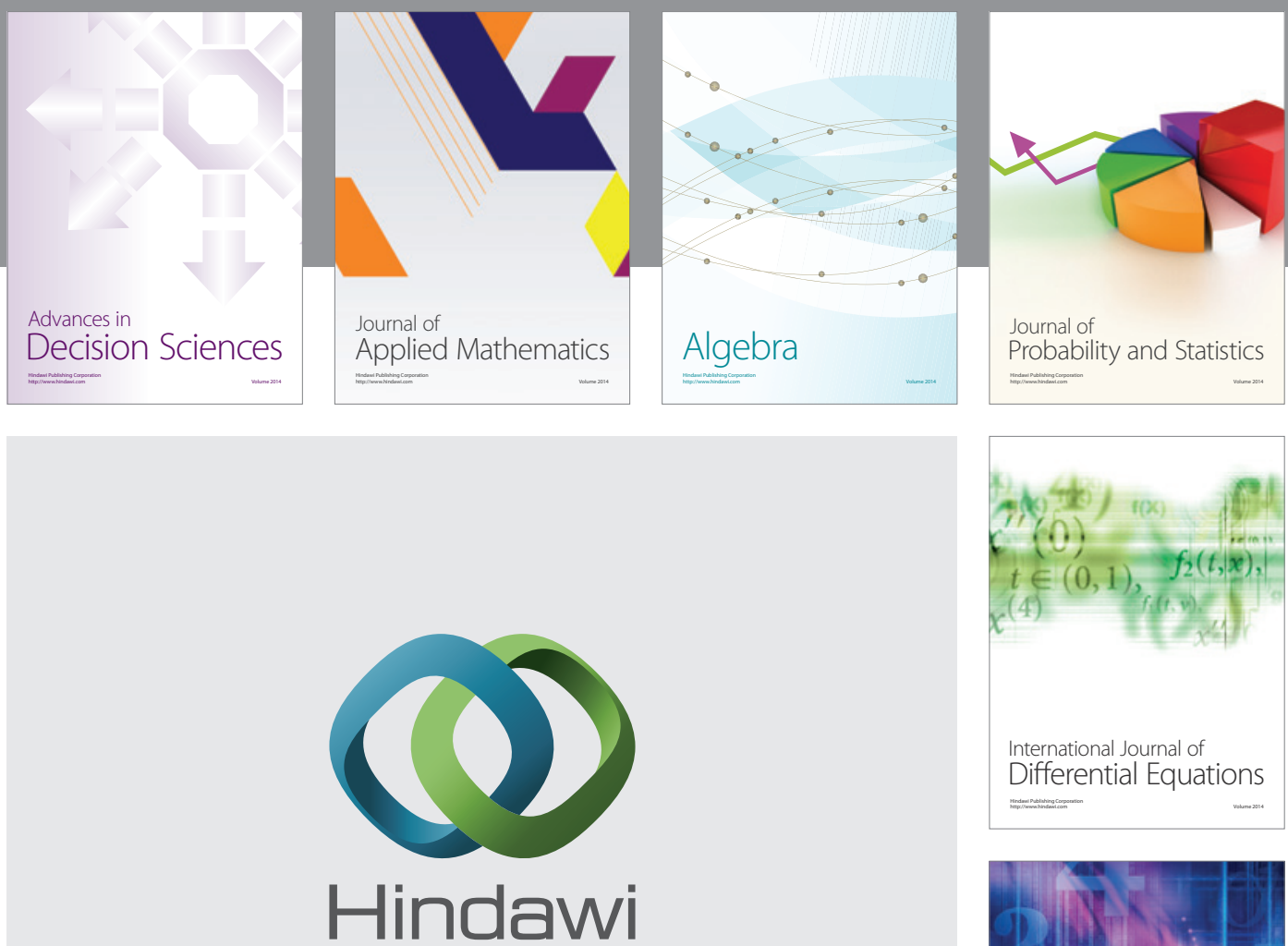

Submit your manuscripts at http://www.hindawi.com
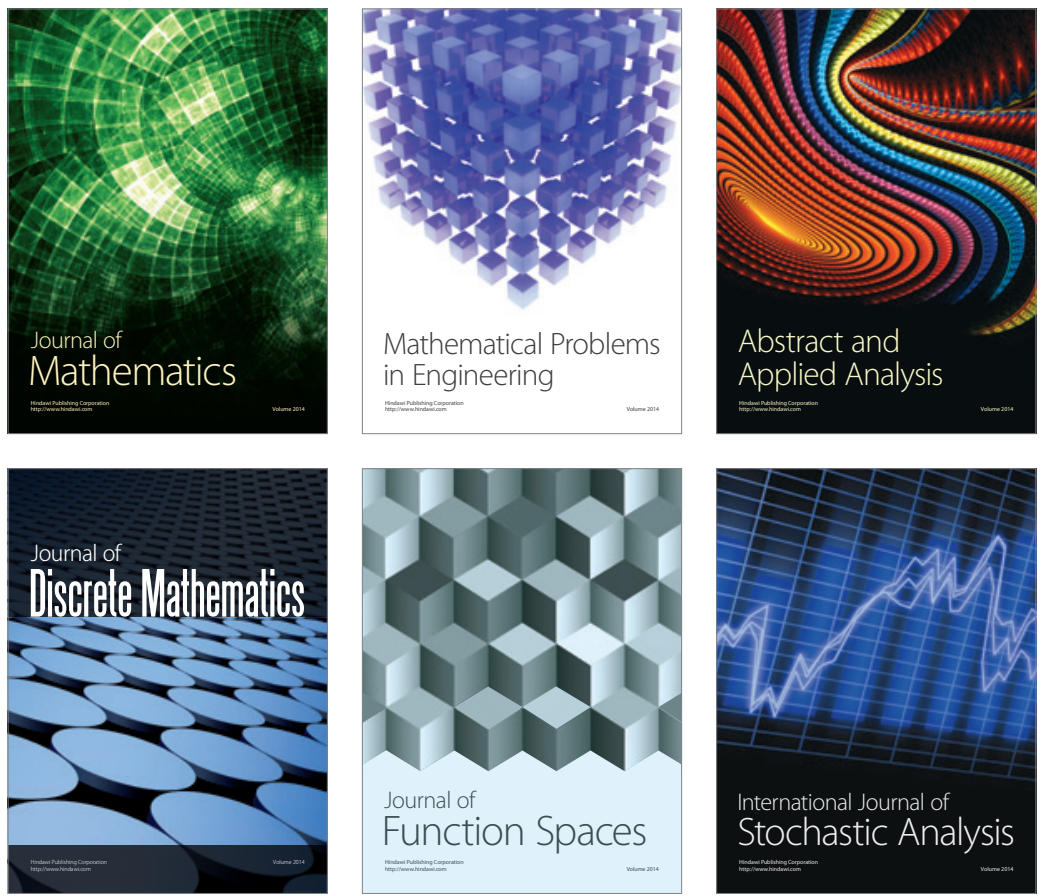

Journal of

Function Spaces

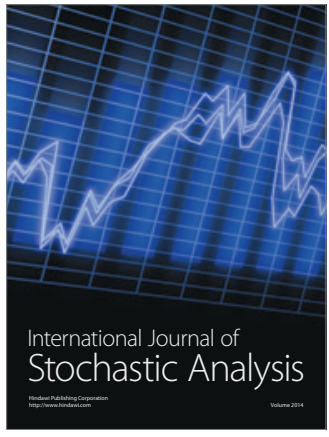

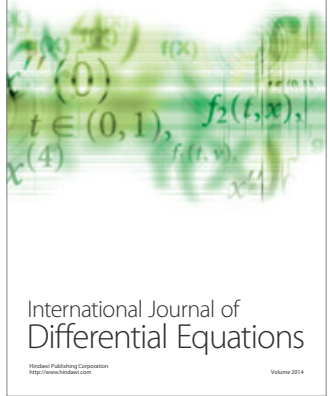
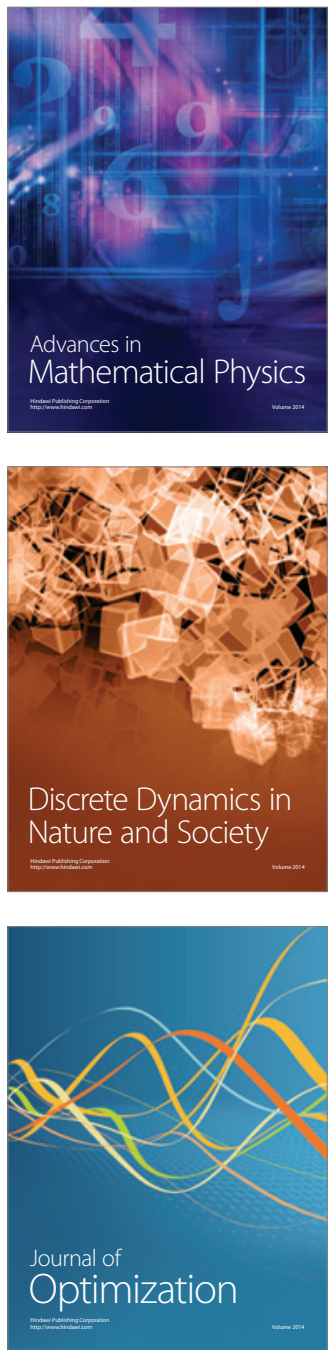